\section{Besser pflegen}

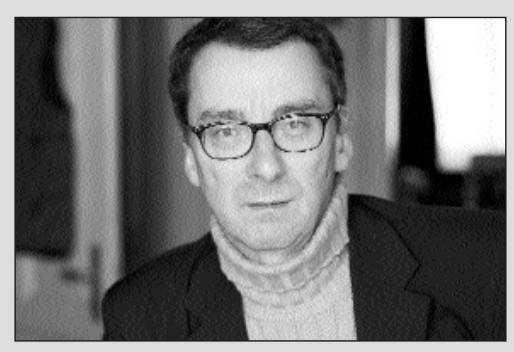

Die Beiträge zum Schwerpunktthema in diesem Heft beleuchten die Situation der Pflege alter Menschen in Deutschland unter verschiedenen Gesichtspunkten.

Herbert Mauel vom Bundesverband privater Anbieter sozialer Dienste würdigt die bisherigen Leistungen der viel gescholtenen fünften Säule der gesetzlichen Sozialversicherung: Die Pflegeversicherung hat viele Menschen von der Sozialhilfe unabhängig gemacht und beim Aufbau einer pflegerischen Infrastruktur mit ambulanten, teilstationären und vollstationären Diensten und Einrichtungen entscheidend mitgeholfen (Seite 12). Prof. Dr. Roland Schmidt von der Fachhochschule Erfurt richtet einen wissenschaftlichen Blick darauf, was auch nach der Koalitionseinigung noch im Argen liegt bei der Pflege. So gibt es beispielsweise immer noch viel zu wenig praktische Modelle, die zeigen, dass es den angeblichen Gegensatz von »ambulant « und »stationär « in der Lebenswelt der Menschen nicht gibt (Seite 17).

Prof. Dr. Heinz Rothgang und Maike Preuss vom Bremer Zentrum für Sozialpolitik zeigen, dass eine Finanzreform in der Pflegeversicherung weiterhin notwendig ist, wenn ein Absenken der realen Pflegeversicherungsleistungen vermieden werden sollen (Seite 21). Und die Pflegesachverständige Anneli Dörfler mag in ihrer kritischen Studie zum Qualitätsmanagement vielen Praktikern aus dem Herzen sprechen: "Es werden immer mehr Daten produziert, die oftmals aus Zeitmangel oder Unkenntnis weder ausgewertet noch analysiert werden. Damit führt sich das Qualitätsmanagement, das auf ständige Verbesserung setzt, selbst ad absurdum." (Seite 25)

Die große Koalition in Berlin hat sich im Juni auf eine Reform der Pflegeversicherung geeinigt. Die wichtigsten Neuregelungen wurden in einem »Eckpunktepapier " festgehalten, das wir in diesem Heft dokumentieren (Seite 14). Der Wortlaut der Gesetzesänderungen und die Umsetzung der Vereinbarungen halten hoffentlich, was sich die Regierungsparteien und auch viele Akteure der Sozialwirtschaft davon vor allem versprechen: bessere Leistungen für pflegebedürftige Menschen, ihre Angehörigen und ihre Helfer.

Gerhard Pfannendörfer - Chefredaktion -

\section{Der „Klassiker“}

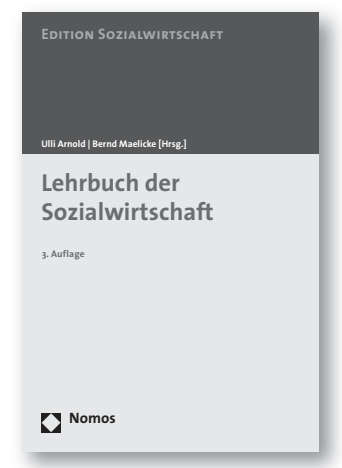

\section{Lehrbuch für}

\section{Sozialwirtschaft}

Von Prof. Dr. Dr. h.c. Ulli Arnold, Universität Stuttgart und Prof. Dr. Bernd Maelicke, Universität Lüneburg

3. Auflage 2007 , ca. 600 S., brosch., ca. 39,- $€$, ISBN 978-3-8329-2680-9

Erscheint Herbst 2007

Die rapide Veränderung der politischen und strukturellen Rahmenbedingungen für die Sozialwirtschaft erfordert grundlegende Änderungen in der Ausbildung und Weiterbildung von Führungskräften sozialer Dienstleistungs-Organisationen.

Die vollständig aktualisierte und neu bearbeitete 3 . Auflage vermittelt Basiswissen und aktuelle Informationen zu den wichtigsten Themen:

- Entwicklung der sozialpolitischen Voraussetzungen

- Anbieter, Wettbewerber, Handlungsfelder, Dienstleistungen

- Typologie und Rechtsformen sozialwirtschaftlicher Organisationen

- Qualitätsmanagement, Sozialmarketing

- Finanzierungsmanagement, Fundraising

- Grundlagen des Managements in der Sozialwirtschaft

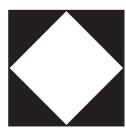

Nomos 